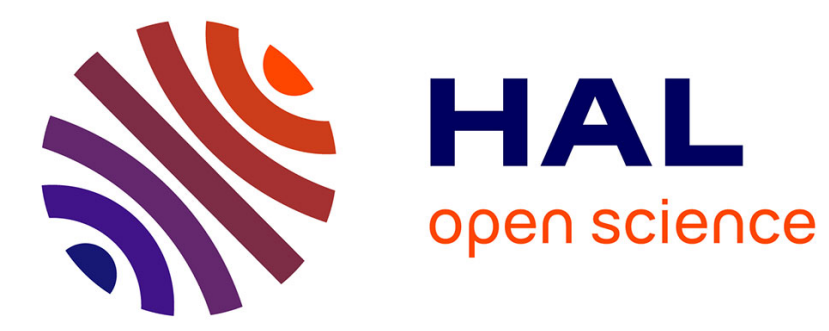

\title{
Fringe Disappearance in Self-Mixing Interferometry Laser Sensors: Model and Application to the Absolute Distance Measurement Scheme Mengkoung Veng, Julien Perchoux, Francis Bony
}

\section{- To cite this version:}

Mengkoung Veng, Julien Perchoux, Francis Bony. Fringe Disappearance in Self-Mixing Interferometry Laser Sensors: Model and Application to the Absolute Distance Measurement Scheme. IEEE Sensors Journal, 2019, 19 (14), pp.5521 - 5528. 10.1109/JSEN.2019.2901125 . hal-02071823

\section{HAL Id: hal-02071823 \\ https://hal.laas.fr/hal-02071823}

Submitted on 18 Mar 2019

HAL is a multi-disciplinary open access archive for the deposit and dissemination of scientific research documents, whether they are published or not. The documents may come from teaching and research institutions in France or abroad, or from public or private research centers.
L'archive ouverte pluridisciplinaire HAL, est destinée au dépôt et à la diffusion de documents scientifiques de niveau recherche, publiés ou non, émanant des établissements d'enseignement et de recherche français ou étrangers, des laboratoires publics ou privés. 


\title{
Fringe Disappearance in Self-Mixing Interferometry Laser Sensors: Model and Application to the Absolute Distance Measurement Scheme
}

\author{
Mengkoung Veng, Julien Perchoux, and Francis Bony
}

\begin{abstract}
The fringes disappearance phenomenon in the selfmixing interferometry occurs whenever the external round-trip phase at free-running state is modulated by either external modulation such as external cavity length changes or internal modulation when the laser injection current is modulated with a high back-scattered light power. This phenomenon has been observed by many authors for harmonic motion or vibration application, and more recently in the case of the absolute distance measurement scheme when the laser injection current is modulated in the triangle waveform. However, there are no clear explanations on the fringes disappearance phenomenon and its dependence on the self-mixing's regimes. In this paper, a novel approach that depicts the mechanism of interferometric fringes disappearance is proposed that highlight with a new perspective the impact of the coupling strength between the laser and the external cavity on the number of missing fringes. The proposed modelling is validated by a set of measurements using the optical feedback interferometry absolute distance measurement scheme.
\end{abstract}

Index Terms-Self-mixing, laser feedback interferometry, fringe disappearance, absolute distance, distance measurement, laser feedback level, laser sensors, interferometry.

\section{INTRODUCTION}

$\mathbf{S}$ ELF-MIXING Interferometry (SMI) (also called Optical Feedback Interferometry) has been studied extensively in the last five decades [1], [2] in various sensing applications such as vibration [3], [4], absolute distance [5], [6], velocimetry [7], [8], micro-scale flow monitoring [9], several biomedical purposes [10], [11] and acoustic pressure imaging [12]. Sensors under the SMI technique have the laser diode as the light source, the interferometer, and the detector. The light from the laser diode propagates towards a distant target where it is partially reflected or back-scattered before being re-injected into the active cavity of the laser. When the laser diode experiences the external optical feedback, the reflected light imprinted with information from the distant target or from the external cavity medium induces perturbation to the operating parameters of the laser such as gain, optical power, lasing frequency and the terminal voltage of the laser [2], [13]. For SMI measurement sensors such as harmonic motion

Manuscript received $<$ DATE $>$; revised $<$ DATE $>$; accepted $<$ DATE $>$; Date of current version $<$ DATE $>$.

M. Veng, J. Perchoux and F. Bony are with the Centre National de la Recherche Scientifique, Laboratory for Analysis and Architecture of Systems, F-31400 Toulouse, France (email:mveng@laas.fr;julien.perchoux@enseeiht.fr;francis.bony@enseeiht.fr).

Color versions of one or more of the figures in this paper are available online at $<$ Link $>$.

Digital Object Identifier $<$ Number $>$ and absolute distance applications, the fringe counting method is basically used to determine the target's displacement and distance respectively.

Two different approaches to modelling the SMI phenomenon have been developed: the three mirror cavity [14] and the perturbation of the rate equation [15]. For sensing applications purpose, the rate equation model is most often invoked and considering the frequency domain of these applications; the rate equations are simplified under the steadystate conditions. Such approximation can be made when the temporal changes in the stimulus are slow relative to the natural frequencies of the laser relaxation frequency and the natural resonant frequency of the external cavity [16]. The single equation that describes the phase condition imposed by the optical feedback is usually referred to as the excess phase equation and is applicable to single-mode lasers [17].

One of the most important and most useful parameter in the excess phase equation is the feedback parameter $C$ as it can be used to qualitatively categorize the regime of the laser under optical feedback [16], [18], [19], [20]. When the feedback level $C \leq 1$, the laser behaviour is stable. On the other hand, when the feedback level $C>1$, more complex phenomena are observed such as hysteresis effect, presence of multiple emission frequencies (including the unstable frequencies [16]), apparent splitting of the emission line due to mode hopping [20] and fringe disappearance phenomenon [21].

A well accepted approach in the community describes the regimes of the laser diode under optical feedback, based on the number of excess phase's solutions or the value of feedback parameter $C$ so that: weak feedback $(0.1<C<1$, only one solution), moderate feedback $(1<C<4.6$, up to three solutions) and strong feedback $(C>4.6$, more than five solutions) [1], [21], [22], [23], [24], [25], [26]. The feedback parameter $C$ is directly involved in the interferometric fringe disappearance phenomenon, and Bernal et al [27] works have described that this phenomenon depends on the regimes described above, i.e., fringes start disappearing only in the strong feedback regime, while $\mathrm{Yu}$ et al [28] demonstrated that the number of fringes is divided by 2 in region $2(7.8<C<14.0), 3$ in region 3 $(14.0<C<20.3)$ and so on. Other publications proposed that two pairs of interferometric fringes for a complete period of modulation disappear when there is a variation of $C$ by $2 \pi$ [29], [30]. However, to the best of our knowledge, no accurate explanations or theories on the mechanism of this phenomenon have been published so far.

The literature mentioned above on interferometric fringe 
disappearance always treated the phenomenon based on the observation made for harmonic motion displacement applications. The core issue in this configuration is the unfeasibility to maintain a constant feedback level $C$ over the target course due mostly to speckle or imperfect alignment. In a recent paper, we reported the observation of the fringe disappearance in the absolute distance measurement scheme [31].

The fringe disappearance phenomenon in SMI measurement sensors will have the significant effect on SMI measurement sensors. As a result, the fringe counting methods will not always be accurate when the laser is operated in $C>1$.

In this paper, we develop the theory describing how interferometric fringes disappear in SMI laser sensors. An absolute distance measurement has been set where the laser diode is operated with modulation of the injection current. As compared to the vibration sensing scheme, the absolute distance approach guarantees a stable feedback parameter $C$ thus allowing for more repeatable experimental conditions.

The paper is organized as follows. Section 2 introduces the relevant self-mixing theory, absolute distance measurement under self-mixing technique, the process of solving the excess phase equation under the moderate/strong feedback and the theory of the fringes disappearance. The experimental setup for validating the theoretical analysis and the experimental results are described and discussed in section 3. The final section, section 4 , concludes this paper.

\section{THEORY}

\section{A. Self-Mixing Interferometry Theory}

The classic representation of the laser diode cavity under feedback is depicted in Fig. 1. The laser diode is the internal cavity with length $L_{\text {in }}$, refractive index $n_{\text {in }}$ and internal roundtrip propagation time $\tau_{\text {in }}$. The mirror $M_{1}$ at the back and $M_{2}$ at the front have the reflectivities (in amplitude) $r_{1}$ and $r_{2}$ respectively. Light leaves the internal cavity through the mirror $M_{2}$ and travels the external cavity of physical length $L_{\text {ext }}$ and effective refractive index $n_{\text {ext }}$ to the surface of the target which is regarded as the third mirror $M_{3}$. The external round-trip propagation time is $\tau_{\text {ext }}$ and the ratio between the emitted light field amplitude and the re-injected light field amplitude is $r_{\text {ext }}$. The laser emission frequency at the free-running state

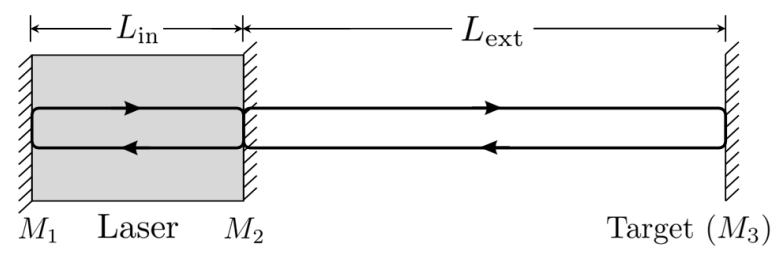

Fig. 1. Schematic of the laser under optical feedback. Solid line with arrows indicates the beam light direction both in the internal and external cavity.

$v_{0}$ is slightly modified to the perturbed lasing frequency $v$ due to the presence of the target leading to the well known excess phase equation [17],

$$
\phi-\phi_{0}+C \sin (\phi+\arctan \alpha)=0,
$$

where $\phi=2 \pi \nu \tau_{\text {ext }}$ represents the external round-trip phase at the perturbed laser called the phase response and $\phi_{0}=$ $2 \pi v_{0} \tau_{\text {ext }}$ represents the external round-trip phase at the freerunning state of the laser called the phase stimulus. $C$ is the feedback parameter and $\alpha$ is the linewidth enhancement factor of laser. The feedback parameter $C$ depends simultaneously on the round-trip time of flight through the external cavity $\tau_{\text {ext }}$ (i.e. the target distance $L_{\text {ext }}$ ), the coupling strength between the target and the laser cavity $\kappa_{\text {ext }}$ and the linewidth enhancement factor $\alpha$ and it is expressed as

$$
C=\frac{\tau_{\text {ext }}}{\tau_{\text {in }}} \kappa_{\text {ext }} \sqrt{1+\alpha^{2}},
$$

where the coupling strength $\kappa_{\text {ext }}$ is defined by the amplitude reflectivity of the laser facets and the external cavity $r_{2}$ and $r_{\text {ext }}$ respectively and the fraction of the reflected light coupled back coherently into the lasing mode $\varepsilon$ [2]. So that:

$$
\kappa_{\mathrm{ext}}=\varepsilon \frac{r_{\mathrm{ext}}}{r_{2}}\left(1-\left|r_{2}\right|^{2}\right) .
$$

The excess phase equation is at the heart of the SMI sensing scheme as it impacts the laser threshold gain which in turn impacts the power emitted by the laser diode [17]

$$
P=\beta \cos \phi,
$$

where $\beta$ is the observable SMI power modulation amplitude.

\section{B. Absolute Distance Measurement}

The distance to the fixed target can be measured by modulating the lasing frequency through the modulation of the laser diode injection current [21], [32]. The relationship of modulation between the emission frequency of the laser power without optical feedback and the injection current is usually considered to be linear which is not entirely correct in practice because of the thermal effect in the laser diode, and several methods have been used to improve the accuracy [5], [33]. A parameter known as the frequency modulation coefficient $\Omega$ is introduced to model this effect, and the phase stimulus $\phi_{0}$ is modified as

$$
\phi_{0}(t)=2 \pi \tau_{\text {ext }}\left[v_{0}+\Omega \Delta I(t)\right]
$$

where $\Delta I$ is the peak to peak amplitude of the modulating current in triangle waveform. With a fixed amplitude at a given frequency modulation $f_{\mathrm{m}}$ under the optical feedback, the output power results in a triangle waveform with small ripples along the ramp which can be expressed as

$$
P(t)^{\prime}=P(t)+\Delta P(t)
$$

where $\Delta P$ is power fluctuation in triangle waveform caused by the side effect of the laser current modulation. Filtering low frequencies in the sensor output signal, it results in a series of sharp peaks which are used to calculate the target distance by simply counting the number $N_{\mathrm{f}}$ of observable peaks (interferometric fringes) [2], [32],

$$
L_{\mathrm{ext}}=\frac{c}{4 \Omega \Delta I n_{\mathrm{ext}}} N_{\mathrm{f}}
$$

where $c$ is the speed of light in vacuum. 


\section{Phase Condition}

In SMI applications sensors, the external round-trip phase at free-running state changes over time because of the modulated operation current of the laser or the change of the external round-trip propagation time $\tau_{\text {ext }}$. The parameters $C$ and $\phi_{0}$ in the excess phase equation (1) are impacted and drive changes to the phase response $\phi$ while the linewidth enhancement factor $\alpha$ is usually considered as constant and its value was reported in the range from $3-7$ [14].

When the feedback parameter $C$ is less or equal to one, the excess phase equation has a unique solution. The relationship between the phase stimulus and the phase response is a simple monotonic function. Fig. 2 shows that the solution of the

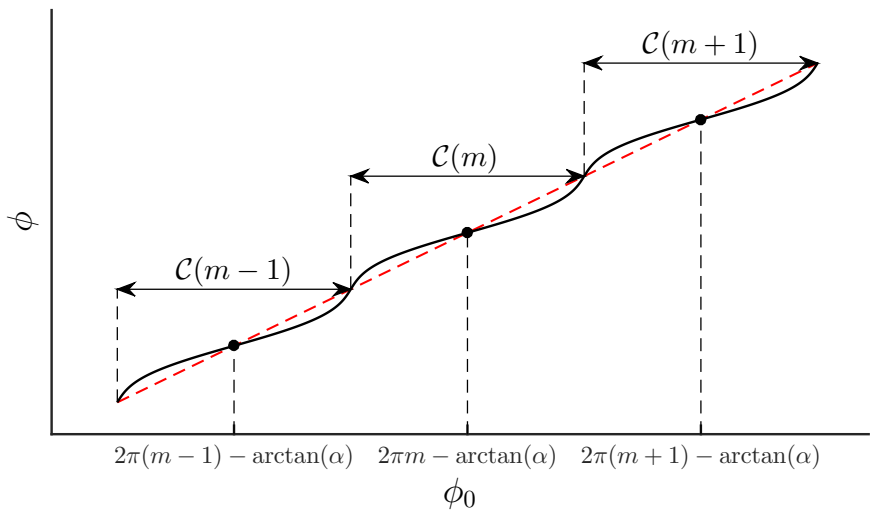

Fig. 2. Plot of the excess phase equation for $C=0.7$, and $\alpha=5$. The red broken line is the axis where there is no optical feedback, and the black solid line indicates the solution path of $\phi$.

excess phase equation are segments of curves $\mathcal{C}(m)$ where $m$ is an integer. Each segment is symmetrical to the point where $\phi_{0}=\phi=2 \pi m-\arctan \alpha$ [34].

When the feedback parameter $C$ is greater than one, the laser under optical feedback is considered operating in moderate or strong feedback, multiple solutions can be found. The relation between $\phi$ and $\phi_{0}$ becomes more complex but it is still maintaining the same symmetrical point for each curve.

Fig. 3 shows that when the feedback parameter $C$ is greater than one, the stable solutions (solid line) are wider and their boundaries can be calculated as being zero of the first derivative of excess phase equation,

$$
\begin{aligned}
0 & =\frac{d}{d \phi}\left[\phi-\phi_{0}+C \sin (\phi+\arctan \alpha)\right] \\
& =1+C \cos (\phi+\arctan \alpha) .
\end{aligned}
$$

By using the sign of the second derivative at the solution to identify low and high boundaries of the stable solutions, we obtain

$\phi_{\mathrm{L}}=(2 m-1) \pi+\arccos (1 / C)-\arctan \alpha \quad$ the low boundary,

$\phi_{\mathrm{H}}=(2 m+1) \pi-\arccos (1 / C)-\arctan \alpha \quad$ the high boundary,

with corresponding value of the phase stimulus

$$
\phi_{0, \mathrm{~L}}=(2 m-1) \pi+\arccos (1 / C)-\arctan \alpha-\sqrt{C^{2}-1},
$$

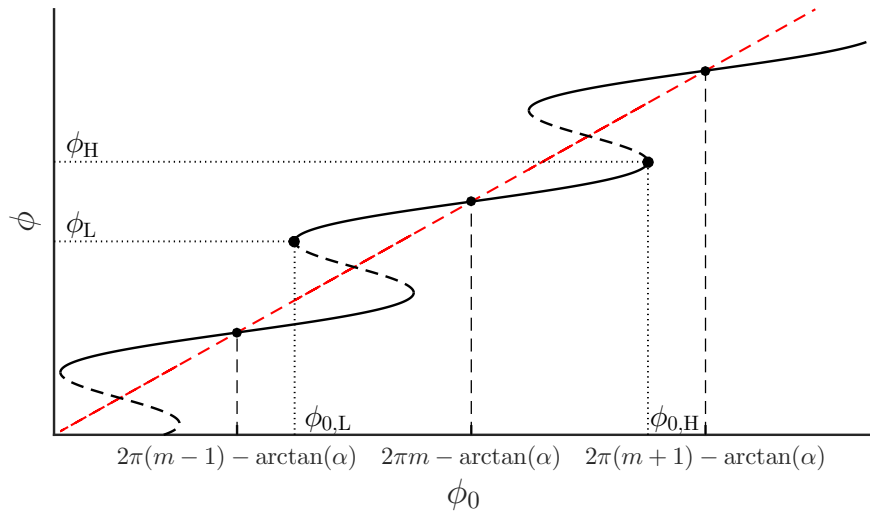

Fig. 3. Plot of the excess phase equation for $C=3$, and $\alpha=$ 5. The red broken line is the axis where there is no optical feedback, and the black solid and broken lines indicate the region of stable and unstable solution of $\phi$ respectively.

$$
\phi_{0, \mathrm{H}}=(2 m+1) \pi-\arccos (1 / C)-\arctan \alpha+\sqrt{C^{2}-1} .
$$

Thus stable solutions segments length of the curve are function of the feedback parameter $C$ as

$$
\phi_{0, \mathrm{H}}-\phi_{0, \mathrm{~L}}=2 \pi-2 \arccos (1 / C)+2 \sqrt{C^{2}-1},
$$

from which it can be observed that the stable solution range is increasing with feedback parameter $C$.

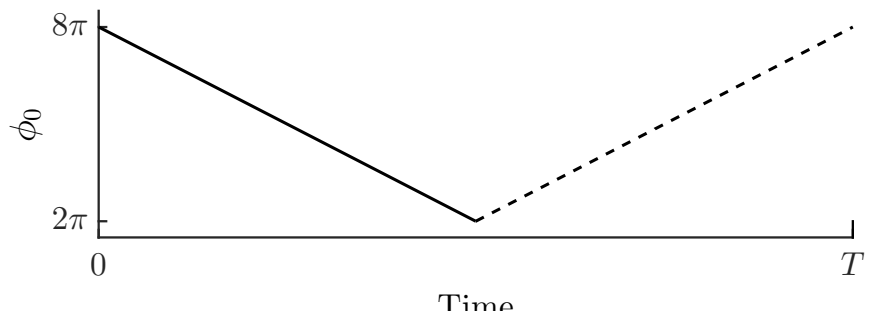

(a)

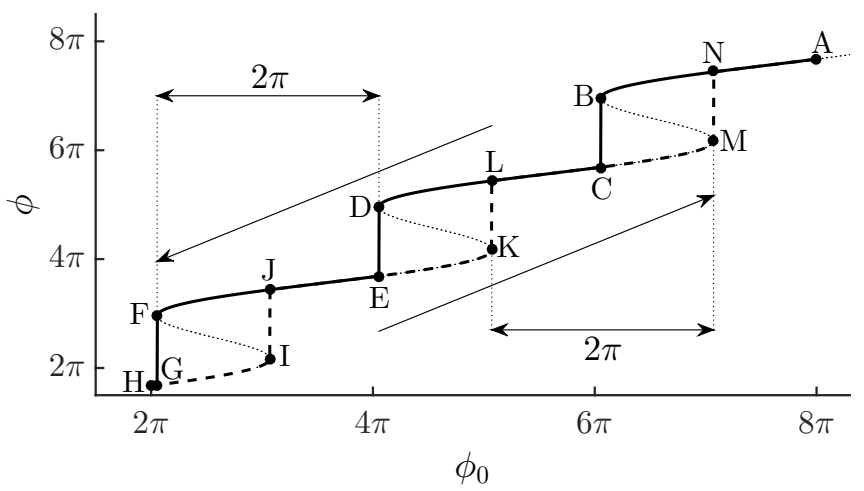

(b)

Fig. 4. Plot (a) show the phase stimulus $\phi_{0}$ is modulated in triangle waveform with a period $T$ and plot (b) is the resulting of phase response $\phi$ with the change of $\phi_{0}$ from $8 \pi$ to $2 \pi$, the feedback parameter $C=3$, and $\alpha=5$. The thin dotted lines shows the unstable solutions to the excess phase equation, the thick solid and dotted lines trace the locus of solution in plot (b) to the phase stimulus in plot (a). 


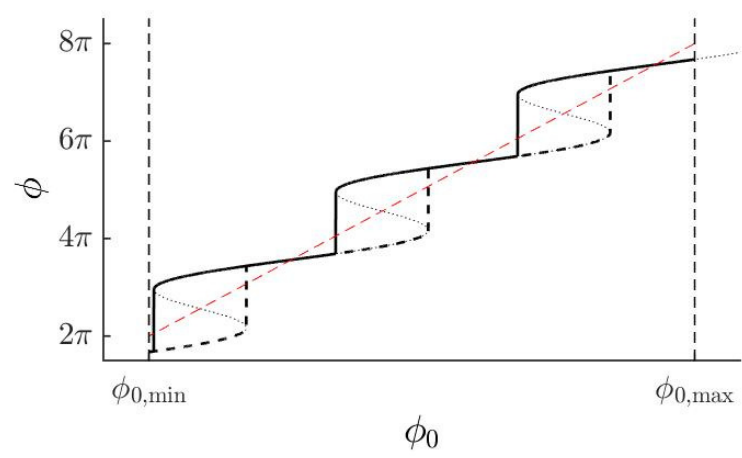

(a)

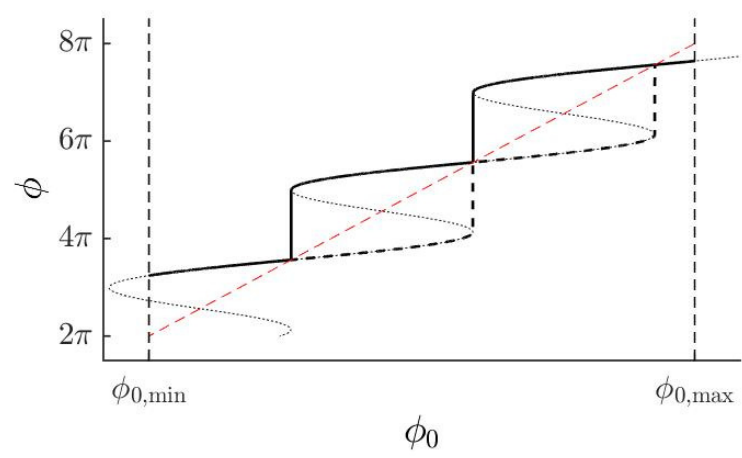

(c)

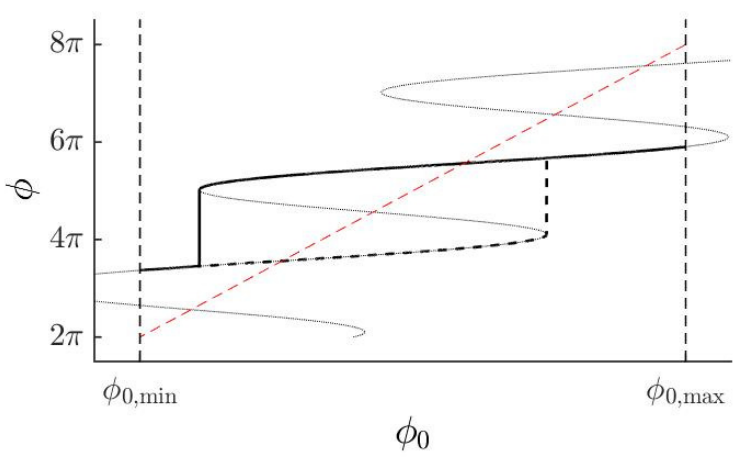

(e)

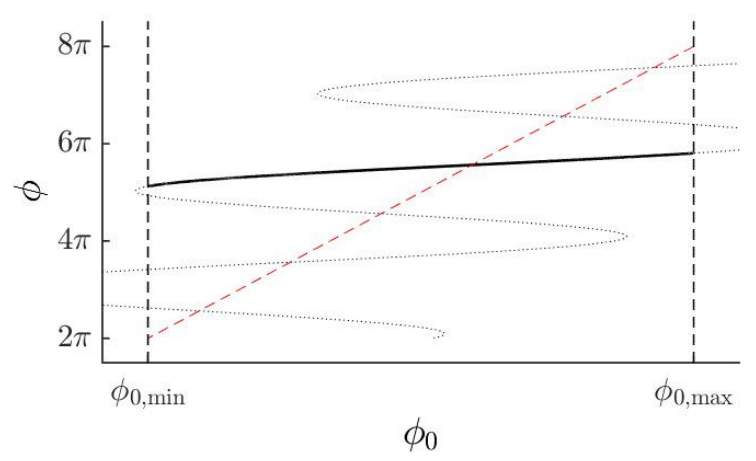

(g)

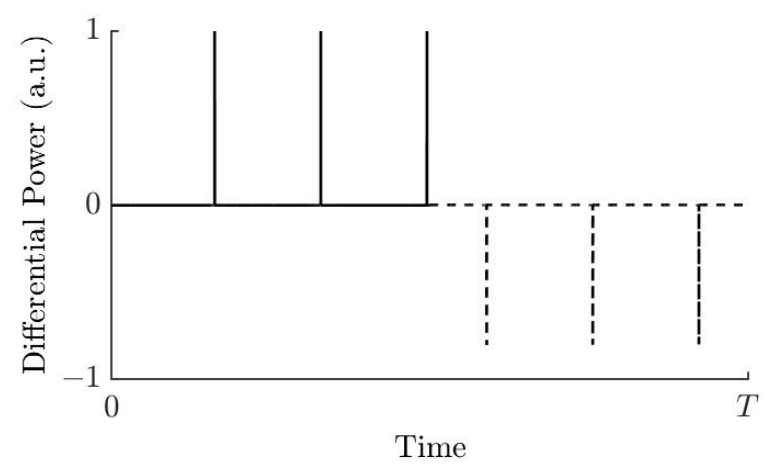

(b)

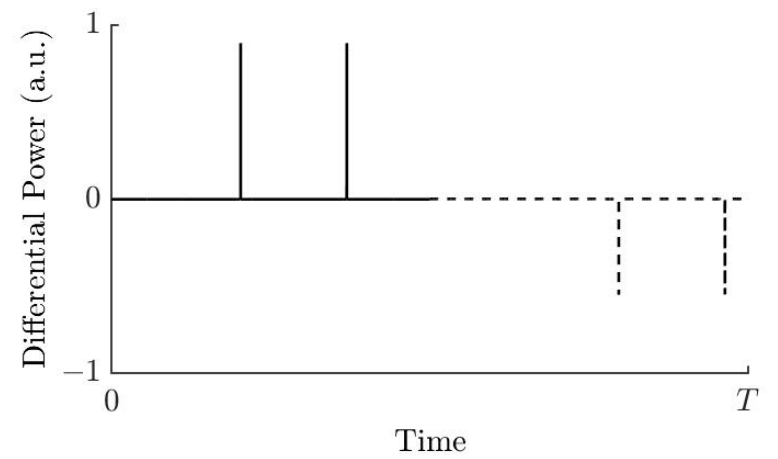

(d)

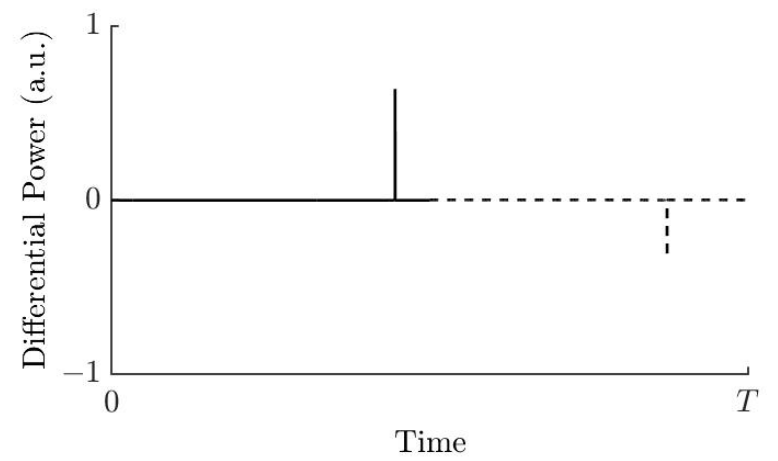

(f)

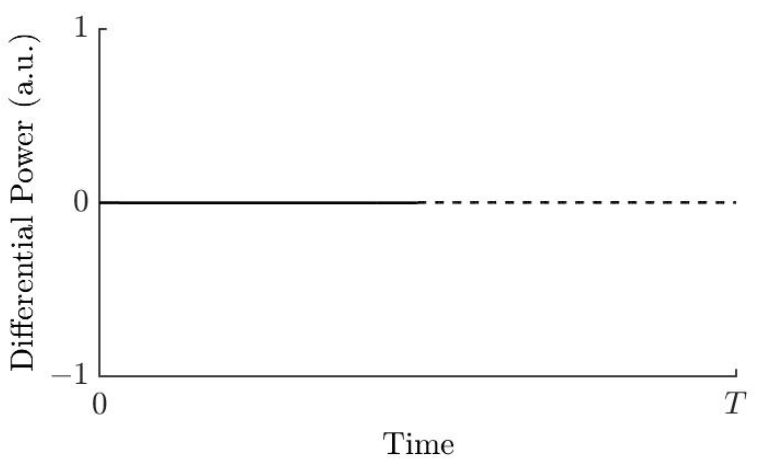

(h)

Fig. 5. Absolute distance measurement with $6 \pi$ phase stimulus's modulation amplitude: (a), (c), (e), (g) are the phase behaviours under different feedback parameters $C ;(\mathbf{b}),(\mathbf{d}),(\mathbf{f})$, and $(\mathbf{h})$ are the results of differential power under different feedback parameters $C$ which correspond to $(\mathbf{a}),(\mathbf{c}),(\mathbf{e}),(\mathbf{g})$ respectively. (a) and (b) are plotted with $C=3$; (c) and (d) are plotted with $C=4.6 ;(\mathbf{e})$ and (f) are plotted with $C=7.5 ;(\mathbf{g})$ and $(\mathbf{h})$ are plotted with $C=10$. 
Fig. 4 illustrates the laser phase behaviour in the case of a triangular modulation of the stimulus phase with an amplitude of $6 \pi$-between $2 \pi$ and $8 \pi$ (Fig. $4(\mathbf{a})$ ) when the parameter $C>1$. The solution to the excess phase equation and physical behaviour are then plotted in Fig. 4(b).

The laser phase starts dwelling solution from point $\mathrm{A}$ to $\mathrm{B}$ when the phase stimulus decreases, then jumps to next closet solution region $\mathrm{C}$ and continues to $\mathrm{D}-\mathrm{E}-\mathrm{F}-\mathrm{G}$ and $\mathrm{H}$. At $\mathrm{H}$, the minimum value of the phase stimulus $\left(\phi_{0}=2 \pi\right)$ is reached. Then the phase stimulus increases, the laser phase dwells solution from point $\mathrm{H}$ to $\mathrm{I}$, then jumps to $\mathrm{J}$ and continues with the path $\mathrm{K}-\mathrm{L}-\mathrm{M}-\mathrm{N}$ and back to $\mathrm{A}$ at the maximum value of the phase stimulus $\left(\phi_{0}=8 \pi\right)$.

\section{Fringes Disappearance Mechanism}

The SMI theories and the phase condition presented in sections above II-A-II-C has been discussed in a similar fashion in [17], [34]. In this section, the novelty of the fringe disappearance's mechanism in SMI is presented by using the phase boundary method. An interferometric fringe disappears whenever the low or high boundary of the stable solution exceeds the limit of the phase stimulus's minimum and maximum respectively. Fig. 5 depicts the phenomenon in the case of a phase stimulus triangle modulation with the amplitude of $6 \pi$ (same as for Fig. 4). Figs. 5(c) and 5(d) represent the phase paths and the differential output power for $C=4.6$ whereas the lowest phase boundary corresponds to a phase stimulus is less than the minimum of actual stimulus thus leading to one missing fringe at each ramp of the modulation. The phase paths and differential output power plotted in Figs. 5(e) and 5(f) are computed for a parameter $C=7.5$ where the highest phase boundary of the third stable region exceeds the maximum of the phase stimulus. An other fringe disappears at each ramp of the modulation leaving a single transition over the $6 \pi$ of stimulus modulation. Eventually, for a parameter $C=10$, no more phase transitions and interferometric fringes appear as shown in Figs. 5(g) and 5(h). It is to be highlighted that the absence of fringes is a consequence of a high feedback parameter $C$ combined with a limited phase stimulus range $(6 \pi)$, as with an extended modulation range, fringes would have remained (for example, $12 \pi$ modulation amplitude would have induced three fringes for the same $C=10$ as shown in Figs. 6). Thus the so-called strong optical feedback regime [1] is not literally a laser regime as it depends mostly on the stimulus modulation (i.e., the bias current modulation or the target displacement amplitudes).

Considering the loss of a fringe for a given feedback parameter $C$ at the lower end side of the stimulus $\phi_{0, \mathrm{~L}}\left(m_{\min }+1, C\right)=$ $\phi_{0, \text { min }}$ (respectively at the higher end side $\phi_{0, \mathrm{H}}\left(m_{\max }-1, C\right)=$ $\left.\phi_{0, \max }\right)$, it can be calculated the increment $\Delta C$ of $C$ for which the $m_{\min }+1$ stable solution limit (respectively $m_{\max }-1$ ) will be equal to the stimulus limit $\phi_{0, \mathrm{~L}}\left(m_{\min }+2, C+\Delta C\right)=\phi_{0, \min }$ (respectively $\left.\phi_{0, \mathrm{H}}\left(m_{\max }-2, C+\Delta C\right)=\phi_{0, \max }\right)$. From (11) and (12) both equalities lead to

$\sqrt{(C+\Delta C)^{2}-1}-\arccos \frac{1}{(C+\Delta C)}=\sqrt{C^{2}-1}-\arccos \frac{1}{C}+2 \pi$.

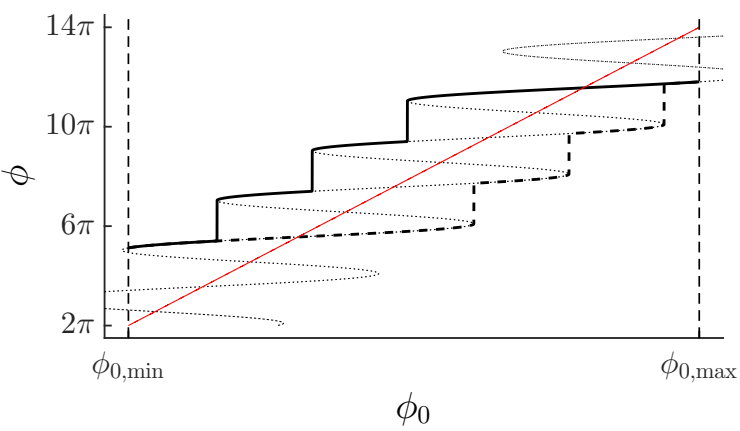

(a) The phase behaviour.

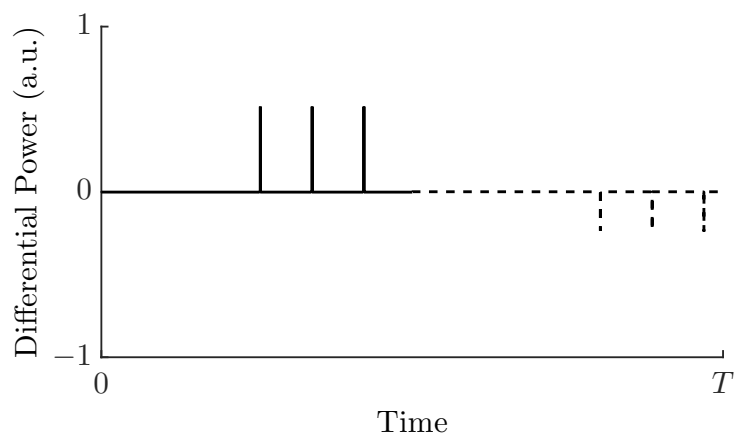

(b) The result of differential power from (a).

Fig. 6. Absolute distance measurement with $12 \pi$ phase stimulus's amplitude modulation under the feedback parameter $C=10$.

It must be highlighted that since fringes are lost alternatively at the lower end and the higher end of the stimulus, the increment of $\Delta C$ results in the loss of two fringes. Fig. 7 shows the graphical solution of equation (14) where it can be observed that $\Delta C$ is a function of $C$ tendentially reaching $2 \pi$ for large $C$ values.

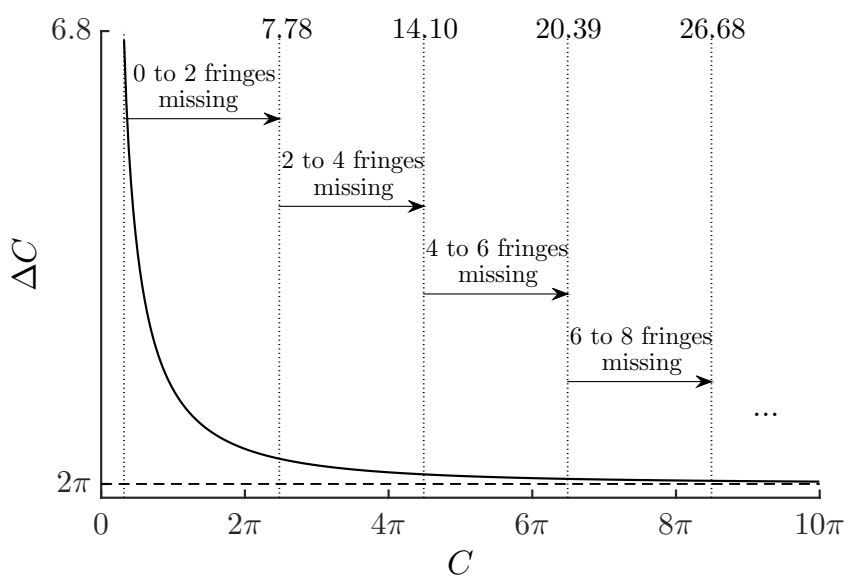

Fig. 7. Evolution of the increment of $C$ that results in the loss of two more fringes as a function of $C$ and definition of the $C$ ranges for which a pair of fringes have disappeared. 


\section{MOdEL VALIDATION}

A block diagram of the experimental setup is depicted in Fig. 8. The DFB laser diode (L1550P5DFB) lasing at $1550 \mathrm{~nm}$ with a package-included monitoring photodiode is associated to a collimating lens which focuses the laser beam onto the target's surface. Two variable optical attenuators (Thorlabs NDC-50S-1 and NDC-50S-3) are displayed along the lasertarget path to control the back-scattered light intensity. A 50/50 beam splitter is used to split the incident light beam from the laser-half goes to a tilt mirror to avoid the reflected light back to the beam splitter and maintain the SMI system in a single cavity, and another haft traverses the distance target to another mirror via the two variable optical attenuators. Then the beam splitter collects $50 \%$ of the reflected light from the target in the front-end detector of a power-meter through a collimating lens. The target is a protected silver mirror (Thorlabs PF10-03-P01) located at a distance of $1.5 \mathrm{~m}$ from the sensor. The laser diode is operated with a bias current of $20 \mathrm{~mA}$ (around 3.34 times its threshold current of $6 \mathrm{~mA}$ ) and modulated with a $5 \mathrm{~mA}$ peak-to-peak amplitude triangle waveform. The photodetected signal then is converted to the voltage by a transimpedance amplifier with a gain of $40 \mathrm{~dB}$ and a selective bandwidth ranging from $40 \mathrm{~Hz}$ to $300 \mathrm{kHz}$ thus allowing to reject the triangle modulation of frequency $10 \mathrm{~Hz}$.

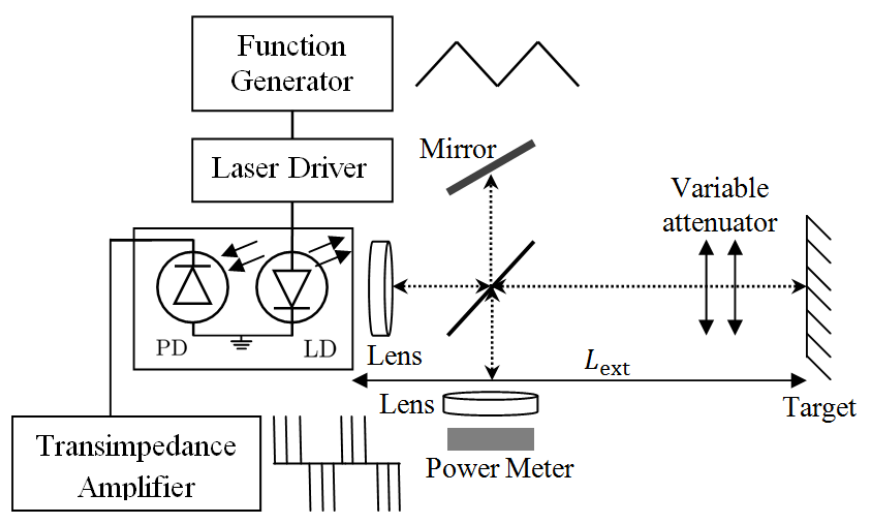

Fig. 8. Block diagram of experimental setup. Laser and photodiode are in the same package.

Prior to the SMI experiment, two parameters are characterized experimentally: the frequency modulation coefficient $\Omega$ and the amplitude reflectivity of the back-scattered light $r_{\text {ext }}$ for the different attenuation conditions in the external cavity. The frequency modulation coefficient is estimated through the experimental results of counting the interferometric fringes in the weak feedback regime, and it results $-430 \mathrm{MHz} / \mathrm{mA}$ at $10 \mathrm{~Hz}$ of frequency modulation. In this experiment, the variation of injection current produces an assumed linear variation in the laser operating frequency; and the thermal effect distortions [5], [33] can be neglected at such low frequency.

The two variable optical attenuators propose eight different attenuation values resulting in sixty-four different feedback levels. Without any attenuators, the amplitude reflectivity coefficient $r_{\text {ext }}$ is $30.12 \%$. The lowest amplitude reflectivity is $0.24 \%$, and twenty-one interferometric fringes per ramp of the triangle modulation are observed. The output power keeps resulting with twenty-one fringes with the target's amplitude reflectivity up to $1.79 \%$. When the amplitude reflectivity is $2.37 \%$, a pair of fringes disappears, then another pair of fringes disappears for a reflectivity of $2.97 \%$. The fringes keep on disappearing as the reflectivity increases, and once it reaches to $25.54 \%$, all the fringes are lost.

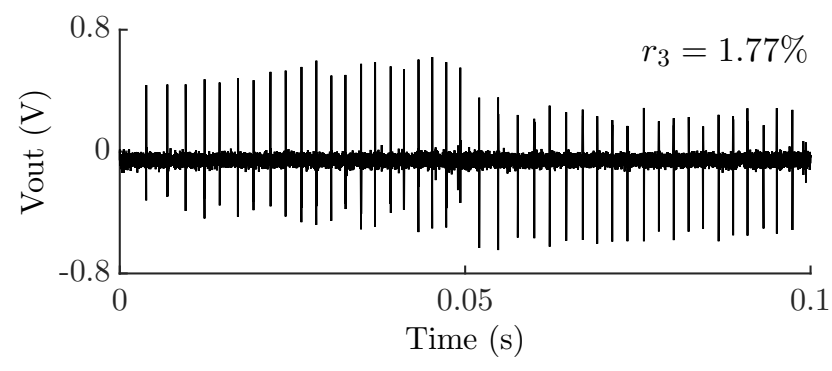

(a)

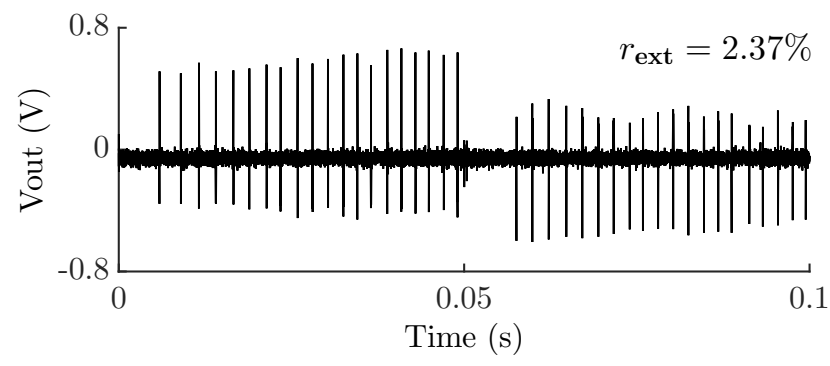

(b)

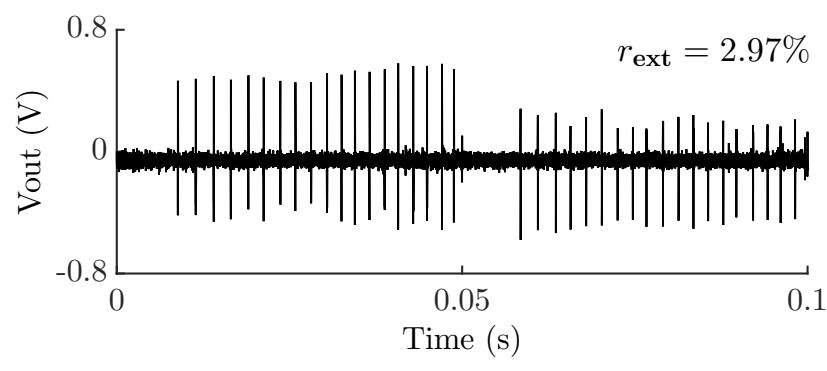

(c)

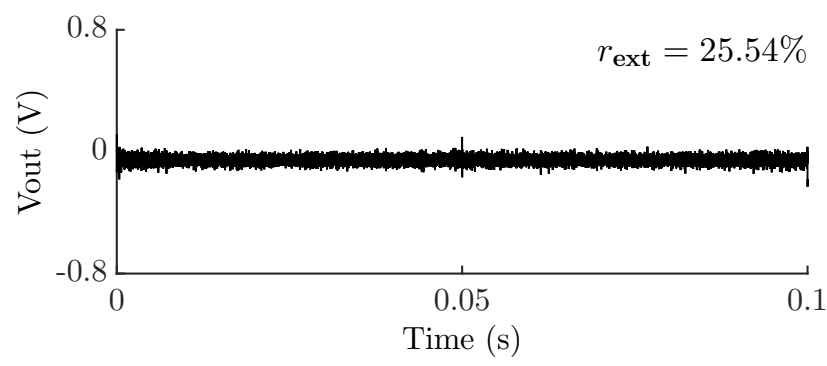

(d)

Fig. 9. Experimental SMI signal acquisition for the distance of $1.5 \mathrm{~m}$ with different target's amplitude reflectivities.

Fig. 9 shows some experimental results with different reflectivities: $1.77 \%, 2.37 \%, 2.97 \%$ and $25.54 \%$. The experiment in Fig. 9(a) shows twenty-one interferometric fringes at the output power with $1.77 \%$ of amplitude reflectivity while for the different reflectivities (Figs. 9(b), 9(c), and 9(d)), the number of fringes decreases. 


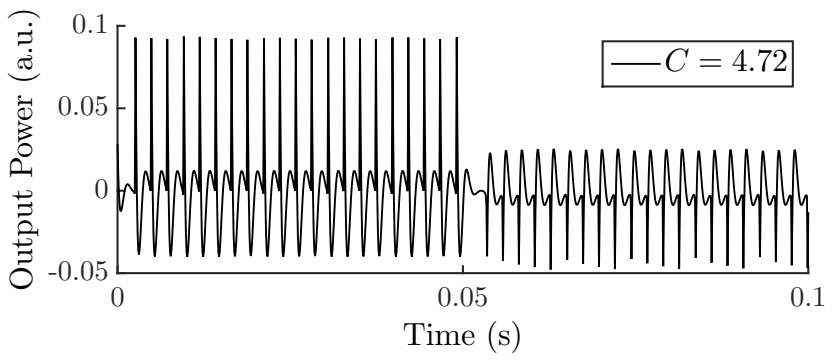

(a)

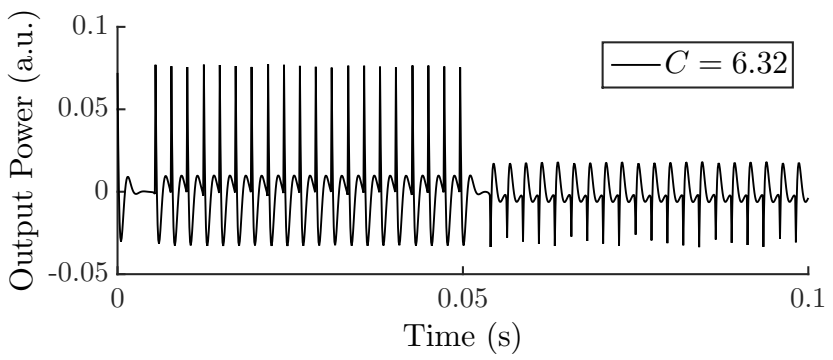

(b)

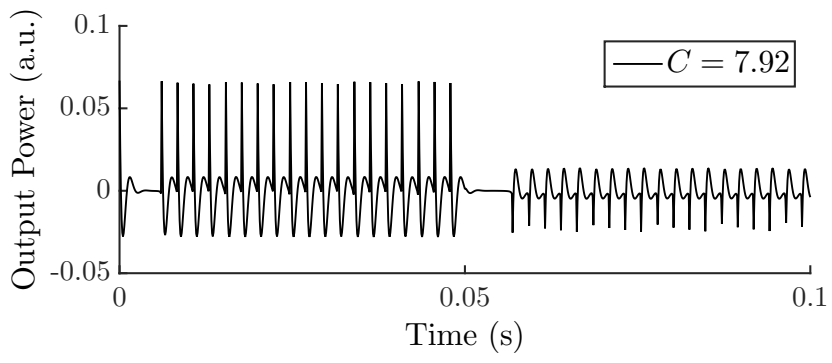

(c)

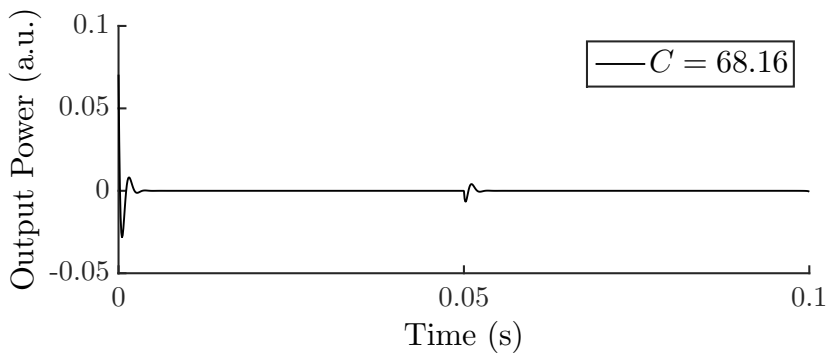

(d)

Fig. 10. Simulation SMI output power in time for the distance of $1.5 \mathrm{~m}$ with different target's amplitude reflectivities.

Using expressions (1) to (6) with realistic intrinsic parameters (laser facet reflectivity $r_{2}$, internal round-trip propagation time $\tau_{\text {in }}$ and linewidth enhancement factor $\alpha$ [35]), the reinjection loss factor $\varepsilon$, with the values measured for the FM coefficient and the reflectivities $r_{\text {ext }}$ and the MATLAB scripts, Fig. 10 shows the simulation of the differential laser power for one period of modulation. The simulation results show a good agreement with the experimental results in particular as concerning the number of missing fringes in each case.

In order to validate the expression in (14), the evolution of the interferometric fringes disappearance as a function of the feedback parameter $C$ is plotted in Fig. 11, both experimentally and theoretically taking optimized value $C=4.84$ and $C=$
7.44 as the value where the first and the second fringes would have disappeared. As can be observed, the model depicted by (14) is in great agreement with the observed experimental results, and thus they validate the model and the explanation of fringe disappearance exposed in this paper.

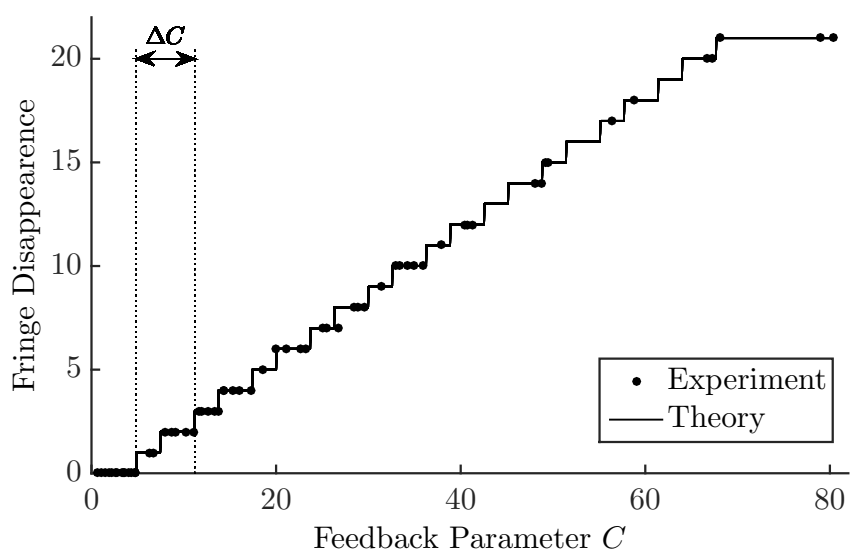

Fig. 11. The behaviour of the interferometric fringe disappearance and the feedback parameter $C$. The thin solid lines show the result of interferometric fringes disappearance in theory and the marker points shows the results of the experiment.

\section{Conclusions}

The present paper describes the mechanism of interferometric fringes disappearance in Self-Mixing Interferometry sensors. This phenomenon is highly dependent on the feedback parameter $C$, and it is described in detail based on the coupled cavity model. The primary cause for fringes disappearance is demonstrated to be the expansion of the excess phase equation stable solutions range with the increment of the parameter $C$, thus reducing the number of stable solutions for a given phase stimulus. This new approach in the modelling of the fringe disappearance phenomenon allows determination of the $C$ values for which a pair of fringes are expected to disappear and as a consequence correlates the number of missing fringes to the value of $C$. This approach is validated both by a behavioural model of the laser under optical feedback and by a series of measurements in the SMI absolute distance configuration.

\section{REFERENCES}

[1] S. Donati, "Developing self-mixing interferometry for instrumentation and measurements," Laser \& Photonics Reviews, vol. 6, no. 3, pp. 393-417. [Online]. Available: https://onlinelibrary.wiley.com/doi/abs/10.1002/lpor.201100002

[2] T. Taimre, M. Nikolić, K. Bertling, Y. L. Lim, T. Bosch, and A. D. Rakić, "Laser feedback interferometry: a tutorial on the self-mixing effect for coherent sensing," Adv. Opt. Photon., vol. 7, no. 3, pp. 570-631, Sep 2015. [Online]. Available: http://aop.osa.org/abstract.cfm?URI=aop-7-3570

[3] N. Servagent, F. Gouaux, and T. Bosch, "Measurements of displacement using the self-mixing interference in a laser diode," Journal of Optics, vol. 29, no. 3, p. 168, 1998. [Online]. Available: http://stacks.iop.org/0150-536X/29/i=3/a=012

[4] Y. Gao, Y. Yu, J. Xi, and Q. Guo, "Simultaneous measurement of vibration and parameters of a semiconductor laser using self-mixing interferometry," Appl. Opt., vol. 53, no. 19, pp. 4256-4263, Jul 2014. [Online]. Available: http://ao.osa.org/abstract.cfm?URI=ao-53-19-4256 
[5] F. Gouaux, N. Servagent, and T. Bosch, "Absolute distance measurement with an optical feedback interferometer," Appl. Opt., vol. 37, no. 28, pp. 6684-6689, Oct 1998. [Online]. Available: http://ao.osa.org/abstract.cfm?URI=ao-37-28-6684

[6] M. Norgia, A. Magnani, and A. Pesatori, "High resolution self-mixing laser rangefinder," Review of Scientific Instruments, vol. 83, no. 4, p. 045113, 2012. [Online]. Available: https://doi.org/10.1063/1.3703311

[7] M. J. Rudd, "A laser doppler velocimeter employing the laser as a mixer-oscillator," Journal of Physics E: Scientific Instruments, vol. 1, no. 7, p. 723, 1968. [Online]. Available: http://stacks.iop.org/0022$3735 / 1 / \mathrm{i}=7 / \mathrm{a}=305$

[8] G. Plantier, N. Servagent, T. Bosch, and A. Sourice, "Real-time tracking of time-varying velocity using a self-mixing laser diode," IEEE Transactions on Instrumentation and Measurement, vol. 53, no. 1, pp. 109-115, Feb 2004.

[9] L. Campagnolo, M. Nikolić, J. Perchoux, Y. L. Lim, K. Bertling, K. Loubière, L. Prat, A. D. Rakić, and T. Bosch, "Flow profile measurement in microchannel using the optical feedback interferometry sensing technique," Microfluidics and Nanofluidics, vol. 14, no. 1, pp. 113-119, Jan 2013. [Online]. Available: https://doi.org/10.1007/s10404012-1029-0

[10] J. Perchoux, A. Quotb, R. Atashkhooei, F. J. Azcona, E. E. RamírezMiquet, O. Bernal, A. Jha, A. Luna-Arriaga, C. Yanez, J. Caum, T. Bosch, and S. Royo, "Current developments on optical feedback interferometry as an all-optical sensor for biomedical applications," Sensors, vol. 16, no. 5, 2016

[11] M. Norgia, A. Magnani, D. Melchionni, and A. Pesatori, "Drop measurement system for biomedical application," IEEE Transactions on Instrumentation and Measurement, vol. 64, no. 9, pp. 2513-2517, Sept 2015.

[12] K. Bertling, J. Perchoux, T. Taimre, R. Malkin, D. Robert, A. D. Rakić, and T. Bosch, "Imaging of acoustic fields using optical feedback interferometry," Opt. Express, vol. 22, no. 24, pp. 30346-30356, Dec 2014. [Online]. Available: http://www.opticsexpress.org/abstract.cfm?URI=oe-22-24-30346

[13] R. Kliese, Y. L. Lim, K. Bertling, A. A. A. Bakar, T. Bosch, and A. D. Rakić, "Self-mixing displacement sensing using the junction voltage variation in a gan laser," in 2008 Conference on Optoelectronic and Microelectronic Materials and Devices, July 2008, pp. 23-25.

[14] K. Petermann, Laser Diode Modulation and Noise. Springer Netherlands, OCLC: 851388814. [Online]. Available: http://public.eblib.com/choice/publicfullrecord.aspx?p=3101640

[15] R. Lang and K. Kobayashi, "External optical feedback effects on semiconductor injection laser properties," IEEE Journal of Quantum Electronics, vol. 16, no. 3, pp. 347-355, Mar 1980.

[16] J. Ohtsubo, Semiconductor lasers: stability, instability and chaos, 3rd ed., ser. Springer series in optical sciences. Springer, no. 111, OCLC: ocn796932145.

[17] R. Kliese, T. Taimre, A. A. A. Bakar, Y. L. Lim, K. Bertling, M. Nikolić, J. Perchoux, T. Bosch, and A. D. Rakić, "Solving selfmixing equations for arbitrary feedback levels: a concise algorithm," Appl. Opt., vol. 53, no. 17, pp. 3723-3736, Jun 2014. [Online]. Available: http://ao.osa.org/abstract.cfm?URI=ao-53-17-3723

[18] G. Acket, D. Lenstra, A. D. Boef, and B. Verbeek, "The influence of feedback intensity on longitudinal mode properties and optical noise in index-guided semiconductor lasers," IEEE Journal of Quantum Electronics, vol. 20, no. 10, pp. 1163-1169, October 1984.

[19] R. Tkach and A. Chraplyvy, "Regimes of feedback effects in 1.5 distributed feedback lasers," Journal of Lightwave Technology, vol. 4, no. 11, pp. 1655-1661, Nov 1986.

[20] S. Donati and R. H. Horng, "The diagram of feedback regimes revisited," IEEE Journal of Selected Topics in Quantum Electronics, vol. 19, no. 4, pp. $1500309-1500309$, July 2013.

[21] T. Bosch, C. Bès, L. Scalise, and G. Plantier, "Optical feedback interferometry," in Encyclopedia of Sensors, C. A. Grimes, E. Dickey, and M. Pishko, Eds. American Scientific Publishers, 2006, vol. vol. X, pp. 1-20. [Online]. Available: http://oatao.univ-toulouse.fr/4059/

[22] O. D. Bernal, H. C. Seat, U. Zabit, F. Surre, and T. Bosch, "Robust detection of non-regular interferometric fringes from a self-mixing displacement sensor using bi-wavelet transform," IEEE Sensors Journal, vol. 16, no. 22, pp. 7903-7910, Nov 2016.

[23] M. Norgia and S. Donati, "A displacement-measuring instrument utilizing self-mixing interferometry," IEEE Transactions on Instrumentation and Measurement, vol. 52, no. 6, pp. 1765-1770, Dec 2003.

[24] G. Giuliani, M. Norgia, S. Donati, and T. Bosch, "Laser diode self-mixing technique for sensing applications," Journal of Optics A:
Pure and Applied Optics, vol. 4, no. 6, p. S283, 2002. [Online]. Available: http://stacks.iop.org/1464-4258/4/i=6/a=371

[25] Y. Yu, G. Giuliani, and S. Donati, "Measurement of the linewidth enhancement factor of semiconductor lasers based on the optical feedback self-mixing effect," IEEE Photonics Technology Letters, vol. 16, no. 4 pp. 990-992, April 2004.

[26] Y. Gao, Y. Yu, J. Xi, Q. Guo, J. Tong, and S. Tong, "Improved method for estimation of multiple parameters in self-mixing interferometry," Appl. Opt., vol. 54, no. 10, pp. 2703-2709, Apr 2015. [Online]. Available: http://ao.osa.org/abstract.cfm?URI=ao-54-10-2703

[27] O. D. Bernal, U. Zabit, and T. Bosch, "Classification of laser self-mixing interferometric signal under moderate feedback," Appl. Opt., vol. 53, no. 4, pp. 702-708, Feb 2014. [Online]. Available: http://ao.osa.org/abstract.cfm?URI=ao-53-4-702

[28] Y. Yu, J. Xi, J. F. Chicharo, and T. M. Bosch, "Optical feedback selfmixing interferometry with a large feedback factor $c$ : Behavior studies," IEEE Journal of Quantum Electronics, vol. 45, no. 7, pp. 840-848, July 2009.

[29] U. Zabit, F. Bony, T. Bosch, and A. D. Rakić, "A self-mixing displacement sensor with fringe-loss compensation for harmonic vibrations," IEEE Photonics Technology Letters, vol. 22, no. 6, pp. 410-412, March 2010.

[30] J. El Assad, "Analysis of self-mixing moderate and strong feedback regimes for mechatronics applications," Ph.D. dissertation, INPT, 2008.

[31] M. Veng, J. Perchoux, and F. Bony, "Impact of high coupling factor in absolute distance measurement with self-mixing interferometry," vol. 1, no. 4 , p. $373,2017$.

[32] E. Gagnon and J. F. Rivest, "Laser range imaging using the self-mixing effect in a laser diode," IEEE Transactions on Instrumentation and Measurement, vol. 48, no. 3, pp. 693-699, Jun 1999.

[33] K. Kou, X. Li, L. Li, and H. Xiang, "Injected current reshaping in distance measurement by laser self-mixing interferometry," Appl. Opt., vol. 53, no. 27, pp. 6280-6286, Sep 2014. [Online]. Available: http://ao.osa.org/abstract.cfm?URI=ao-53-27-6280

[34] G. Plantier, C. Bes, and T. Bosch, "Behavioral model of a self-mixing laser diode sensor," IEEE Journal of Quantum Electronics, vol. 41, no. 9, pp. 1157-1167, Sept 2005.

[35] L. A. Coldren, S. W. Corzine, and M. L. Mašanovitć, Diode lasers and photonic integrated circuits. John Wiley \& Sons, 2012, vol. 218.

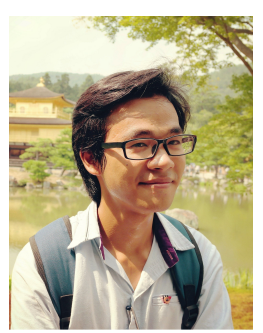

Mengkoung Veng received the engineer degree in electrical and electronics from the Institute of Technology of Cambodia in 2013 and the Master's in Electronic Systems for Embedded and Communicating Applications (ESECA) from ENSEEIHT-INP of Toulouse in 2014. He is currently a Ph.D. student at the Research Group on Optoelectronics for Embedded System (OSE) team of the LAAS-CNRS in Toulouse, France, working on the self-mixing interferometry specifically in absolute distance application.

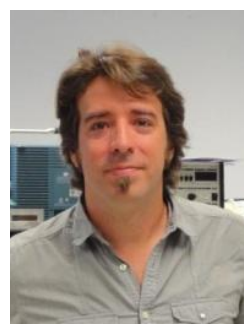

Julien Perchoux was born in Vénissieux, France, in 1977. He received the B.Sc. degree in applied physics from the University of Montpellier, France, in 1999, the M.Sc.degree and the Ph.D. degree in microwave and photonics from the University of Toulouse - ISAE, in 2002 and 2005 respectively. He was graduated from the École Normale Supérieure, Cachan, France in 2001. He is at the head of the Optoelectronics for Embedded System (OSE) team of the LAAS-CNRS (Toulouse, France) and associated professor of the Toulouse INP since 2008. His current research interests include semiconductor laser physics, biomedical imaging, flowmetry, acoustic imaging and design of embedded sensing systems. 


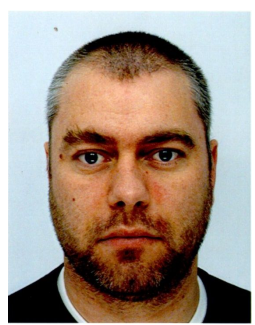

Francis Bony was born in 1975 . He received his $\mathrm{PhD}$ in microelectronics-microsystems in 2003, prepared at the Laboratoire d'Analyse et d'architecture des systèmes of the French Centre National de la Recherche Scientifique (LAAS-CNRS), about infrared sensors. In 2005, he became Assistant Professor in the electronics Laboratory of ENSEEIHT in Toulouse. He is currently working on digital circuits and electronic system boards for optoelectronic measurement sensors. 